ARTICLE

Received 17 Apr 2015 | Accepted 12 Dec 2015 | Published 25 Jan $2016 \quad$ DOl: 10.1038/ncomms10474 OPEN

\title{
Evolutionary signals of selection on cognition from the great tit genome and methylome
}

Veronika N. Laine ${ }^{1, \star}$, Toni I. Gossmann ${ }^{2, \star}$, Kyle M. Schachtschneider ${ }^{3,4, \star}$, Colin J. Garroway ${ }^{5}$, Ole Madsen ${ }^{3}$, Koen J.F. Verhoeven ${ }^{6}$, Victor de Jager ${ }^{1}$, Hendrik-Jan Megens ${ }^{3}$, Wesley C. Warren ${ }^{7}$, Patrick Minx ${ }^{7}$, Richard P.M.A. Crooijmans ${ }^{3}$, Pádraic Corcoran², The Great Tit HapMap Consortium ${ }^{\dagger}$, Ben C. Sheldon ${ }^{5}$, Jon Slate ${ }^{2}$, Kai Zeng ${ }^{2}$, Kees van Oers ${ }^{1}$, Marcel E. Visser ${ }^{1,3} \&$ Martien A.M. Groenen ${ }^{3}$

For over 50 years, the great tit (Parus major) has been a model species for research in evolutionary, ecological and behavioural research; in particular, learning and cognition have been intensively studied. Here, to provide further insight into the molecular mechanisms behind these important traits, we de novo assemble a great tit reference genome and whole-genome re-sequence another 29 individuals from across Europe. We show an overrepresentation of genes related to neuronal functions, learning and cognition in regions under positive selection, as well as increased CpG methylation in these regions. In addition, great tit neuronal non-CpG methylation patterns are very similar to those observed in mammals, suggesting a universal role in neuronal epigenetic regulation which can affect learning-, memory- and experience-induced plasticity. The high-quality great tit genome assembly will play an instrumental role in furthering the integration of ecological, evolutionary, behavioural and genomic approaches in this model species.

\footnotetext{
${ }^{1}$ Department of Animal Ecology, Netherlands Institute of Ecology (NIOO-KNAW), P.O. Box 50, 6700AB Wageningen, The Netherlands. ${ }^{2}$ Department of Animal and Plant Sciences, University of Sheffield, Sheffield S10 2TN, UK. ${ }^{3}$ Animal Breeding and Genomics Centre, Wageningen University, P.O. Box 338, 6700AH Wageningen, The Netherlands. ${ }^{4}$ Department of Animal Sciences, University of Illinois, Urbana, Illinois 61801, USA. ${ }^{5}$ Edward Grey Institute, Department of Zoology, University of Oxford, Oxford OX1 3PS, UK. ${ }^{6}$ Department of Terrestrial Ecology, Netherlands Institute of Ecology (NIOO-KNAW), P.O. Box 50, 6700AB Wageningen, The Netherlands. ${ }^{7}$ The Genome Institute, Washington University School of Medicine, St Louis, Missouri 63108, USA. * These authors contributed equally to this work. $\dagger$ A full list of consortium members appears at the end of the paper. Correspondence and requests for materials should be addressed to V.N.L. (email: v.laine@nioo.knaw.nl) or to M.A.M.G. (email: martien.groenen@wur.nl).
} 
T heory predicts that the ability to perceive, assess and learn from others should have fitness benefits under a wide range of conditions ${ }^{1}$. However, we know little about whether, or how, natural selection acts on cognitive traits related to social living in any species ${ }^{2}$. Great tits learn socially in the wild ${ }^{3}$, solve complex learning tasks ${ }^{4}$ and there is evidence that cognitive abilities may have important fitness implications $s^{5}$. A literature survey of records of technical (for example, tool use) and opportunistic (for example, exploring novel food items) innovation spanning 803 bird species from 76 families shows that great tits are among the top avian species in terms of overall number and diversity of foraging innovations ${ }^{6}$. This suggests rapid evolution in the great tit lineage of learning and cognitionassociated traits compared with many other birds, making great tits an excellent model for studying complex social-cognitive behaviour.

In addition to studies of cognitive and learning abilities of great tits ${ }^{3,5}$, great tit research has contributed significantly to our general understanding of life history evolution ${ }^{7}$, the effects of climate change on natural populations ${ }^{8,9}$, the allocation of resources to breeding ${ }^{10}$ including trade-offs between reproduction and immunity ${ }^{11}$, the extent and consequences of individual variation in rates of ageing ${ }^{12}$, inbreeding and inbreeding depression ${ }^{13,14}$, host-parasite coevolution ${ }^{15}$, territorial and foraging behaviour ${ }^{16,17}$ and the impact of variation in personality traits on other life history characters ${ }^{18}$. This considerable contribution of studies of great tits to our understanding of basic ecological and evolutionary processes is largely due to work on numerous long-term study systems throughout Europe.

To explore the molecular basis for learning and cognition, we developed a complete set of de novo molecular genomic tools to ascertain evidence of natural selection on genetic and epigenetic variation in great tits. Here, we test the hypothesis that learning and cognition have been important targets of selection in great tit evolution by exploring footprints of selection in the great tit genome. We find an overrepresentation of genes related to neuronal functions, learning and cognition in regions under positive selection, as well as increased $\mathrm{CpG}$ methylation in these regions. In addition, great tit neuronal non- $\mathrm{CpG}$ methylation patterns are very similar to those observed in mammals. The development of high-quality genomic resources for great tits provides tools that will allow the integration of genomics into ecological, evolutionary and behavioural research in wild populations in this important study system.

\section{Results}

Genome sequencing, assembly and annotation. We selected a male great tit (hereafter the reference bird) from a recent captive population (four generations in captivity) in the Netherlands for genome sequencing and de novo assembly (see Methods and Supplementary Information for a detailed description of genome assembly and annotation). We generated a total of $114 \mathrm{~Gb}$ of Illumina HiSeq sequence data; after gap-filling and removal of adaptor sequences, the assembly consisted of a total of 2,066 scaffolds with an N50 scaffold length of over 7.7 Mb and an N50 contig length of $133 \mathrm{~kb}$. Taken together, the assembled contigs span $1.0 \mathrm{~Gb}$. We were able to assign $98 \%$ of the assembled bases to a chromosomal location using the recently described highdensity linkage map ${ }^{19}$ (Supplementary Data 1). The total number of chromosomes covered by the assembly is 29 with three additional linkage groups (Chr25LG1, Chr25LG2 and LGE22). The assignment of chromosomal locations to the majority of the assembly resulted in a very high quality reference genome and enabled detailed comparisons with other bird genomes.
A comparison between the genomes of the great tit and zebra finch (Taeniopygia guttata) ${ }^{20}$ (see Supplementary Fig. 1) showed only intra-chromosomal inversions, and not a single interchromosomal rearrangement, confirming high synteny in birds. For the genome annotation step, we combined RNA sequencing data from eight different tissues of the reference bird with gene models from chicken (Gallus gallus) ${ }^{21}$ and zebra finch ${ }^{20}$. The final annotation resulted in a total of 21,057 transcripts for 13,036 high-confidence gene predictions.

Population genomics. To obtain further insight into the evolutionary genetics of the great tit, an additional 29 wild individuals from across Europe were re-sequenced at an average depth of $10 \times$ (Supplementary Data 2, Fig. 1a). We found very little differentiation among populations $\left(F_{\mathrm{ST}}<0.02\right.$; Supplementary Data 3 ) which is in line with previous phylogenetic analyses of the spatial genetic structure of great tits in Europe using mtDNA markers ${ }^{22}$. This suggests a largely panmictic European great tit population $^{22}$. Pairwise sequential Markovian coalescent analysis (PSMC) $)^{23}$ of the reference individual suggested a large effective population size that increased from an already large $\sim 2 \times 10^{5}$ individuals $1 \mathrm{Myr}$ ago to $\sim 5.7 \times 10^{5}$ individuals $70 \mathrm{Kyr}$ ago (Fig. 1b). There was evidence that the expansion was interrupted by a very mild decline in population size beginning $\sim 110 \mathrm{Kyr}$ ago, coinciding with the start of the last glacial period in Europe, followed by a quick recovery. Consistent with expectation for a population that has undergone a recent increase, there is a genome-wide excess of low-frequency variation, as indicated by negative Tajima's $D$ values (Fig. $2 \mathrm{~b}$ ).

Selective sweeps. We found that several regions with reduced diversity coincide with regions of extremely negative Tajima's $D$ (Fig. 2a,b), indicative of recent selective sweeps. To investigate the role of recent positive selection in greater detail, we conducted a genome-wide scan for selective sweeps and identified 813 genomic regions (Fig. 2, Supplementary Data 4) compromising roughly $0.2 \%$ of the genome. We extracted 460 genes that had human orthologues from these regions and assessed their functional importance by performing a gene ontology (GO) enrichment analysis. Thirteen different GO categories were overrepresented in these selected regions relative to the rest of the genome (Fig. 3, Supplementary Data 5a). Among these categories were dendrite, cognition and several other categories related to neuronal function. Therefore genes related to neuronal function are likely to have been targets of recent positive selection.

Learning and cognition. One of the striking genes presented in the learning/cognition category was early growth response protein 1 (EGR1), whose gene expression has been particularly well studied within vocal communication and social contexts in Passerines $^{24,25}$. EGR1 is one of the immediate early genes, which are known to be important in learning and memory ${ }^{26}$. In zebra finches, its expression has been shown to be correlated with song learning and practice and is social context dependent ${ }^{25,27}$. To further investigate the role of EGR1 in great tit evolution, we obtained additional EGR1 sequences from 45 bird species (Supplementary Data 6). We found that rapid evolution of EGR1 is specific to the tit lineage (as signified by an elevated ratio of non-synonymous to synonymous substitution rate $(\mathrm{dN} / \mathrm{dS})$ ) and has occurred after the split with the nearest sequenced relative, the ground tit and that this is not a feature related to the captive nature of the reference bird (Supplementary Fig. 2). These results are consistent with EGR1 being subject to frequent, recurrent positive selection. Another relevant gene from this category was the forkhead box protein P2 (FOXP2), a well-studied gene that 
affects speech and language development in humans ${ }^{28}$ and linked to song learning in birds ${ }^{29,30}$. The combination of these songrelated genes and the other neuronal genes within sweep areas points to a role of these genes in the evolution of song learning and memory in great tits.

Rates of molecular evolution. To examine whether the identified $\mathrm{GO}$ categories in general play an important role in great tit evolution, we extracted all great tit genes that are associated with the enriched GO terms of the sweep regions (Fig. 3, Supplementary Data 5a) and conducted evolutionary rate $(\mathrm{dN} / \mathrm{dS})$ analyses by using orthologous genes from chicken and zebra finch. We found that median $\mathrm{dN} / \mathrm{dS}$ for genes associated with the identified GO terms is reduced compared with all other GO-annotated genes (0.066 versus $0.087, \quad P=1.4 \times 10^{-22}$, Mann-Whitney $U$-test), and yet these genes have significantly more targets of probable positive selection based on a site test of positive selection $\left(P=1.84 \times 10^{-3}, \chi^{2}\right.$ test; see Methods for details). Moreover, on a genome-wide scale, $\sim 1 \%$ of the genes in the great tit genome show evidence for positive selection based on their long-term evolutionary rates. These genes are generally enriched for neuronal traits, such as cerebellar Purkinje cell layer formation and axon extension (Supplementary Data 5b). Taken together, it becomes apparent that selection on traits for brain function has played a major role in the evolution of great tits.

The great tit methylome. Epigenetic control of gene expression is increasingly recognized as playing a major role in many different cellular processes affecting a large variety of traits ${ }^{31}$, with DNA methylation of cytosines being the most widely studied epigenetic mark. Great tit DNA methylation patterns were investigated by performing whole-genome bisulfite sequencing in whole brain and blood tissue of the reference bird. A total of 10.2 million CpG sites, representing $66.7 \%$ of all CpG sites, could be called in both tissues. The observed genome-wide methylation patterns in both tissues, including reduced methylation within CpG islands and at transcription start sites (TSS), are consistent with previous findings in human and mouse cells ${ }^{32,33}$ (Supplementary Fig. 3). We also observed low, but significant non-CpG methylation in the brain tissue that was not observed in the blood (Fig. 4a). The neuronal non-CpG methylation patterns seen at 167.4 million sites ( $42 \%$ of all non-CpG sites) display similar genome-wide patterns to those seen for $\mathrm{CpG}$ methylation, including reduced methylation within CpG islands and at TSS, albeit at a much

a

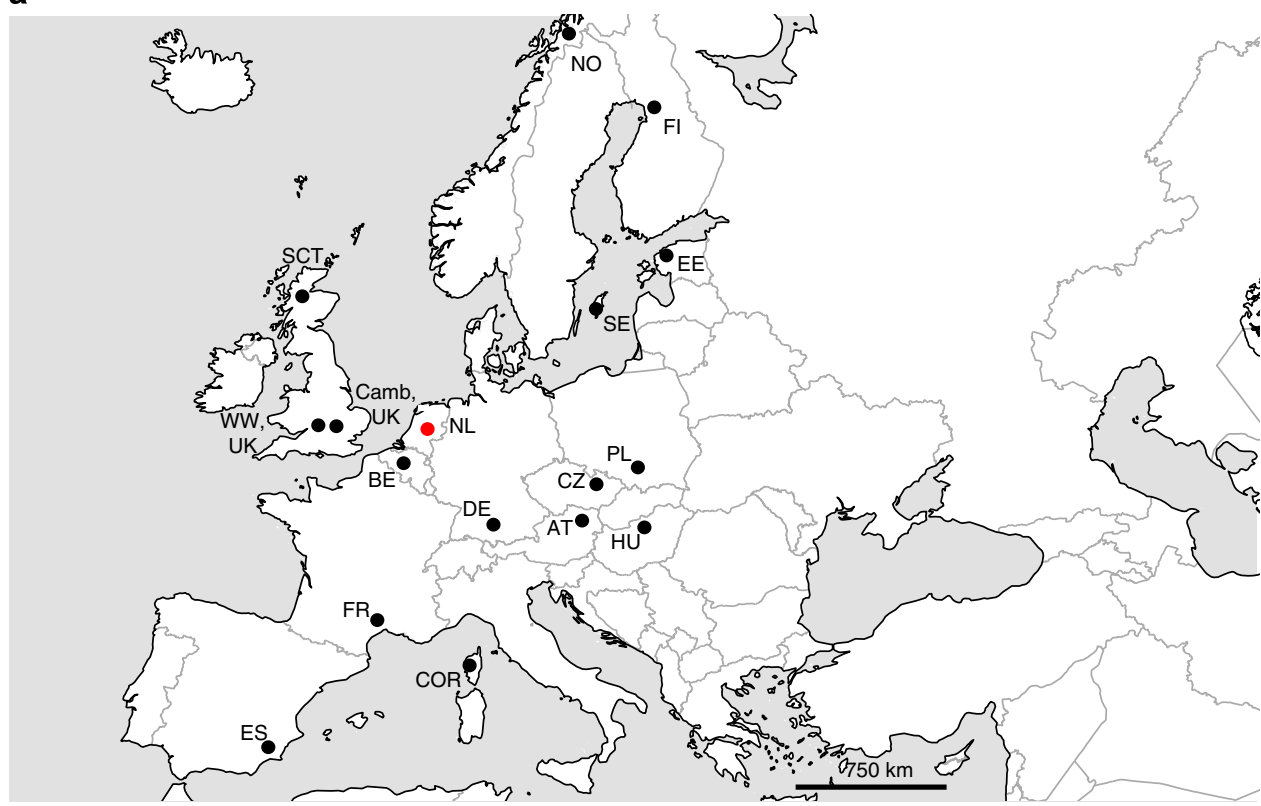

b

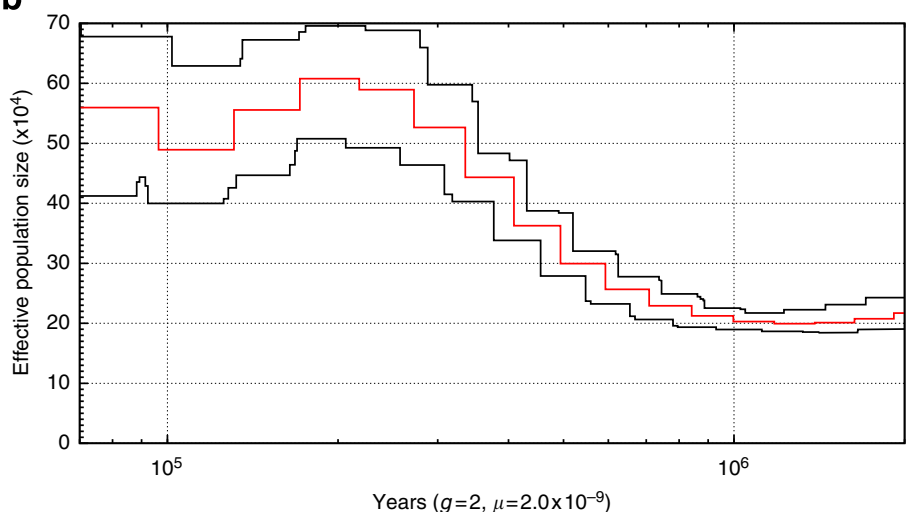

Figure 1 | The 29 re-sequenced great tits and their demographic history. (a) Map of the sampling locations of the 29 re-sequenced great tits (black) and reference individual (red). (b) Pairwise sequential Markovian coalescent analysis (PSMC) of the reference genome. The red line represents the average and the black lines indicate the confidence interval as determined by bootstrapping $(100 \times)$. 


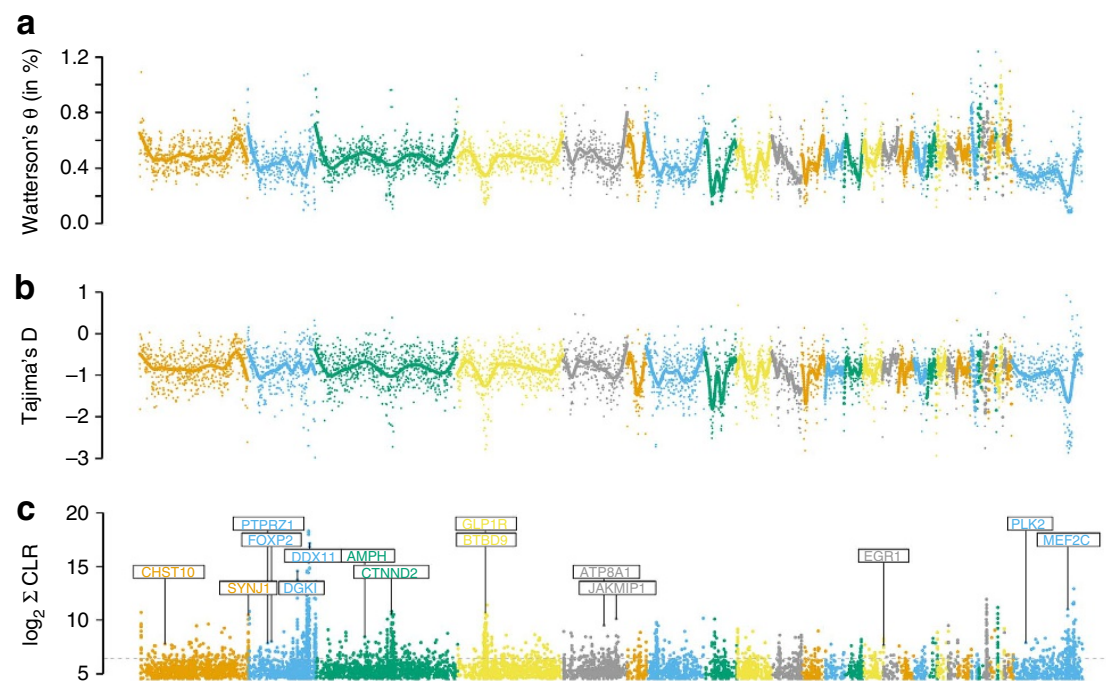

Figure 2 | Genome-wide test statistics obtained from 29 re-sequenced great tits. (a) Genome-wide distribution of Watterson's $\Theta$ and (b) Tajima's D measured in sliding window sizes of $50 \mathrm{kB}$ and step size of $10 \mathrm{kB}$, as well as (c) CLR (composite likelihood score, measured as the sum of neighbouring sweep targets, see Methods) from the sweep analysis with labelled cognition-related genes (see Fig. 3 and Supplementary Data 5a) that were among the top 3\% of gene-associated sweep targets (indicated by the dashed line). Chromosomes are separated by colour in ascending order according to their chromosome number. The $\mathrm{Z}$ chromosome is the furthest right. The solid lines in the upper two panels denote smoothing splines.

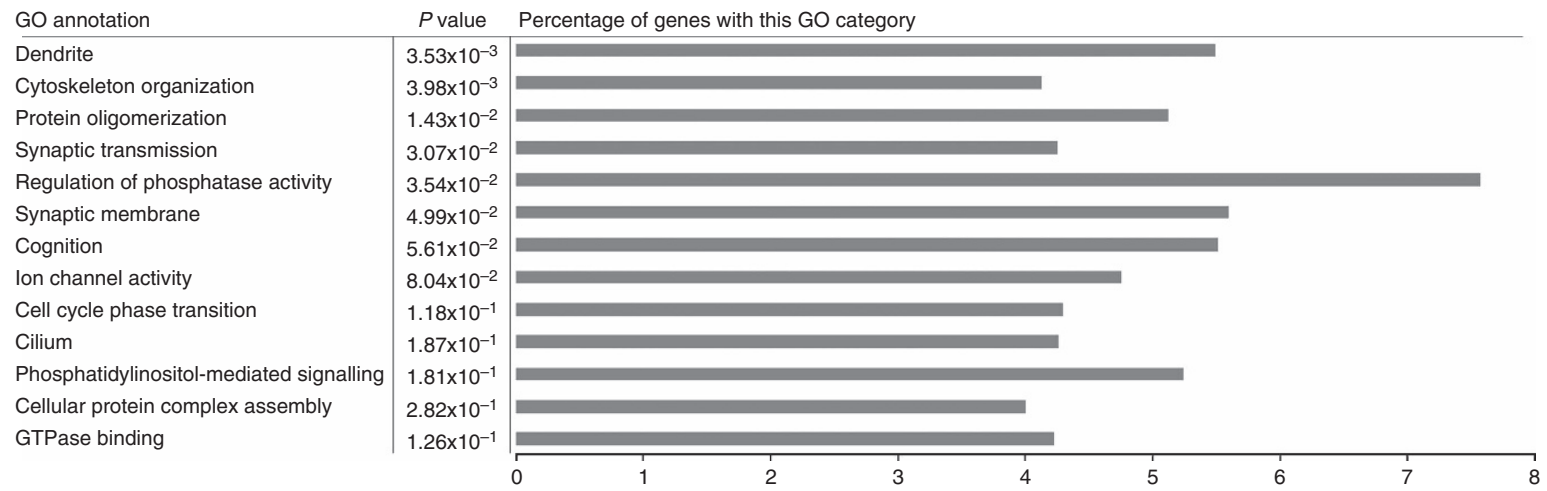

Figure 3 | Gene ontology (GO) enrichment analysis of sweep area genes by using human gene ontologies. The GO enrichment analysis detected 13 functional groups of GO terms across all sweep areas (first column). $P$ value denotes the corrected $P$ value by using Bonferroni step-down method.

lower level. Similar non-CpG methylation has recently been found in embryonic stem cells and oocytes of many mammalian species $^{34,35}$, as well as in the neuronal tissue of humans and mice 33,35 .

Both CpG and non-CpG methylation has been shown to negatively correlate with gene expression at TSS and in gene bodies in mouse neurons ${ }^{33}$, while hypomethylation of both CpG and non-CpG sites in gene bodies has been reported in highly expressed genes of human neurons ${ }^{35}$. Consistent with these results, both $\mathrm{CpG}$ and non-CpG methylation were negatively correlated with gene expression within gene bodies and at TSS in the great tit brain (Spearman's rho $<-0.23, P<1.0 \times 10^{-95}$ for all comparisons, Fig. $4 \mathrm{~b}, \mathrm{c})$. In addition, the negative correlation between non-CpG methylation and expression was observed in the regions directly upstream and downstream of gene bodies (Spearman's rho $<-0.22, P<0.0001$ for all comparisons, Fig. 4c). Therefore, our findings now extend a potential functional role of non- $\mathrm{CpG}$ methylation in neuronal tissue to Aves, suggesting evolutionary conservation of this epigenetic regulation in brains.

Methylation is correlated with rates of molecular evolution. To investigate the potential adaptive and evolutionary role of DNA methylation, we first assessed the methylation patterns of selective sweep genes in the brain. We observed higher CpG methylation at sweep gene bodies (Linear Mixed effect Model, LMM; $\chi_{1}^{2}=394.61, \quad P=9.2 \times 10^{-88}$ ) and in regions upstream $\left(\mathrm{LMM} ; \chi_{1}^{2}=148.94, P=3.0 \times 10^{-34}\right)$ and downstream (LMM; $\left.\chi_{1}^{2}=292.70, P=1.8 \times 10^{-65}\right)$ of gene bodies compared with the same regions in genes outside sweep regions (Fig. 4d). In addition, lower non-CpG methylation in sweep gene bodies $\left(\mathrm{LMM} ; \chi_{1}^{2}=57.17, P=4.0 \times 10^{-14}\right)$ and in regions upstream (LMM; $\quad \chi_{1}^{2}=10.10, \quad P=0.001$ ) and downstream (LMM; $\left.\chi_{1}^{2}=31.88, \quad P=1.64 \times 10^{-8}\right)$ of gene bodies was observed compared with the same regions in genes outside sweep regions (Fig. 4e). These patterns are not due to systematic differences in expression levels between sweep and non-sweep genes because the observed $\mathrm{CpG}$ and non-CpG methylation differences are in opposing directions. In addition, although the overall expression profiles reveal a higher proportion of non-sweep genes with no or low expression (Supplementary Fig. 4e), differences seen between $\mathrm{CpG}$ and non-CpG methylation in sweep gene regions were also observed when comparing genes with similar expression levels (Supplementary Fig. 4a-d). While previous studies in chickens and humans have also shown altered $\mathrm{CpG}$ methylation in regions under selective pressure ${ }^{36}$, it is unclear what causes the observed 

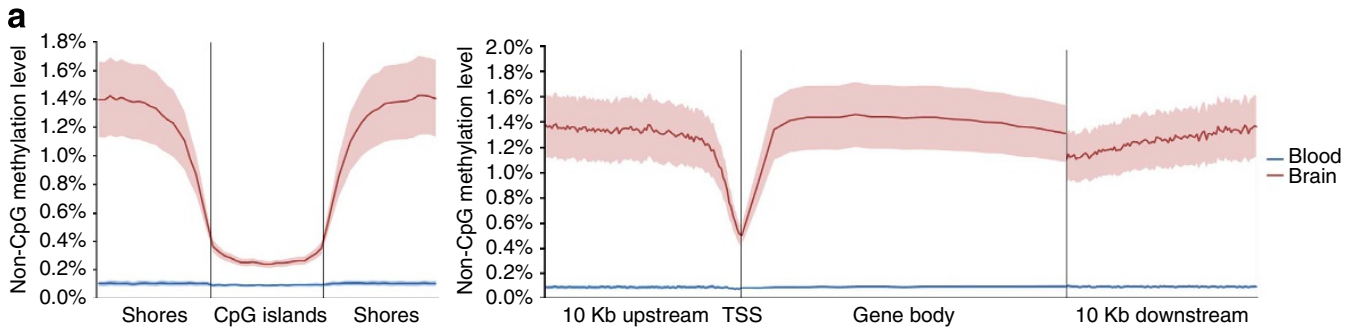

b
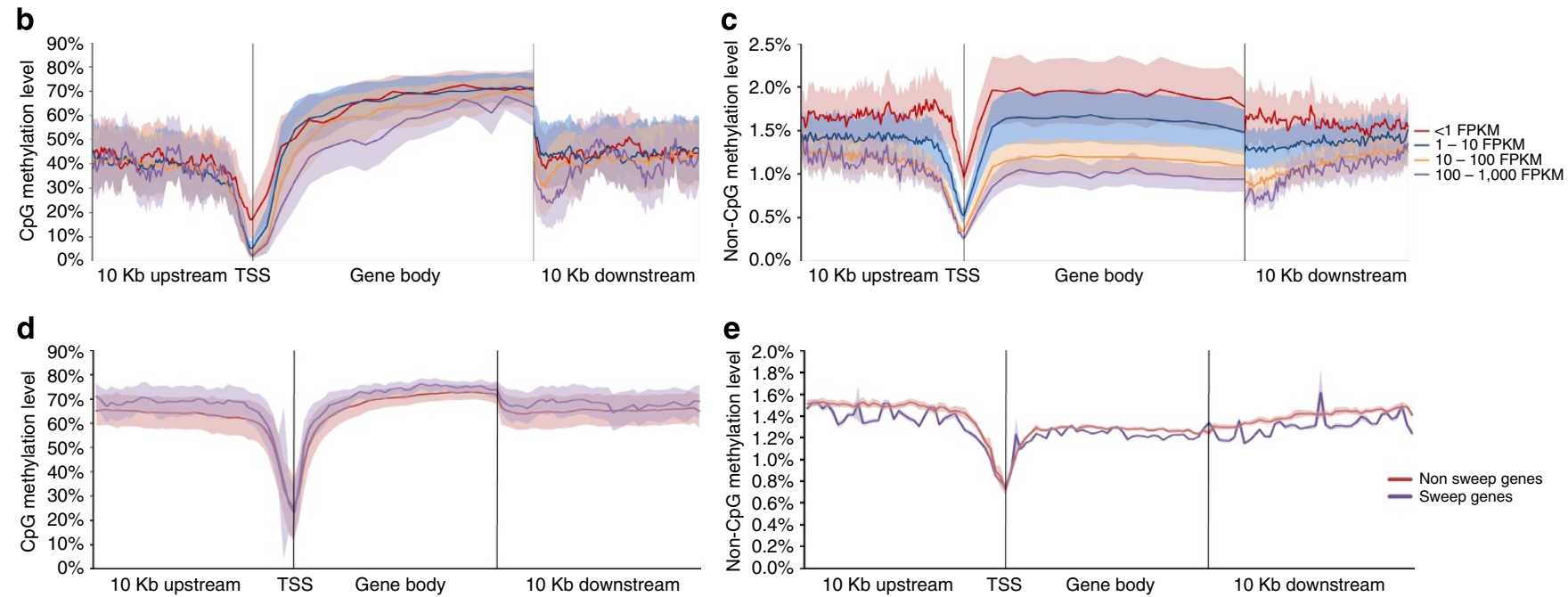

Figure 4 | DNA methylation patterns across genomic features. (a) Non-CpG methylation patterns associated with $\mathrm{CpG}$ islands and gene bodies. (b) Neuronal CpG methylation in gene bodies and at TSS is negatively correlated with expression (Spearman's rank correlation, Spearman's rho < - 0.23 , $P<1.0 \times 10^{-95}$ for all comparisons), presented as fragments per kilobase of transcript per million fragments mapped (FPKM). (c) Neuronal non-CpG methylation at TSS, gene bodies and adjacent upstream and downstream regions is negatively correlated with expression (Spearman's rho < -0.23 , $P<1.0 \times 10^{-95}$ for all comparisons). (d) Increased neuronal CpG methylation at sweep gene bodies (Linear Mixed Effect Model, LMM; $\chi_{1}^{2}=394.61$, $\left.P=9.2 \times 10^{-88}\right)$ and adjacent upstream $\left(\mathrm{LMM} ; \chi_{1}^{2}=148.94, P=3.0 \times 10^{-34}\right)$ and downstream regions $\left(\mathrm{LMM} ; \chi_{1}^{2}=292.70, P=1.8 \times 10^{-65}\right)$.

(e) Decreased neuronal non-CpG methylation in sweep gene bodies $\left(\mathrm{LMM} ; \chi_{1}^{2}=57.17, P=4.0 \times 10^{-14}\right)$ and adjacent upstream (LMM; $\chi_{1}^{2}=10.10$, $P=0.001)$ and downstream regions $\left(L M M ; \chi_{1}^{2}=31.88, P=1.64 \times 10^{-8}\right)$. Shaded areas denote variances.

higher $\mathrm{CpG}$ and lower non-CpG methylation in great tit sweep regions. In addition, lowly methylated genes in the brain were found to evolve significantly slower in comparison with highly methylated genes as shown by differences in the rate of nonsynonymous mutations between the two groups of genes $(P<0.001$ for all pairwise comparisons, $U$-test, Supplementary Fig. 5). This pattern was observed for both $\mathrm{CpG}$ and non-CpG methylation at TSS and within gene bodies. Overall these results not only show, for the first time, conserved neuronal non-CpG methylation patterns between birds and mammals, but also extend them by showing that methylation is correlated with rates of molecular evolution, thereby suggesting an important role for DNA methylation in evolution.

\section{Discussion}

We have generated a high quality de novo assembled and annotated genome for the great tit, a model organism in ecology and evolutionary biology. Our study adds to the growing number of representative genomes sequenced across the bird family tree $\mathrm{e}^{37,38}$. Further, unlike these recent genome studies, we assembled the great tit genome into chromosomes, which was useful for determining chromosomal regions of selective sweeps and larger-scale synteny conservation across species. The great tit genome assembly represents a powerful tool, especially when combined with the extensive availability of individual phenotypes of known birds for which DNA is available via routine blood sampling. Using sequence analysis of birds from a wide range of
European populations, we identified selective sweep areas enriched with genes related to learning and cognition. Using whole-genome methylation data, we not only revealed conserved non-CpG methylation patterns between birds and mammals, but also extended these observations by showing that methylation is correlated with rates of molecular evolution, thereby suggesting an important role for DNA methylation in evolution. Our de novo assembled genome will help us to reveal the genetic basis of phenotypic evolution, which is essential for understanding how wild species have adapted to our changing planet.

\section{Methods}

Genome sequence assembly and annotation. A blood sample from a male Parus major was obtained for whole-genome sequencing and stored in queens buffer. This reference bird originated from a captive population that was derived from wild-caught birds from the Netherlands four generations ago and has since been artificially selected for avian personality ${ }^{39}$. In the great tit genome assembling, we relied on the creation of de Bruijn graphical structures, a directed graph that evolves from defined sequence length (kmer) progression (see Supplementary Methods for a detailed description of genome assembly and annotation). In brief, our genome assembling involved four principal steps that progressed from sequence quality revisions, to forming contigs from these sequence reads, to connecting contigs into scaffolds using paired-end sequence of large fragments (jumping libraries) and finally gap filling. In this study, the total input genome coverage of Illumina HiSeq sequences was $\sim 95 \times$ (fragments, 3 and $8 \mathrm{~kb}$ spanning inserts) based on a genome size estimate of $1.2 \mathrm{~Gb}$. The combined sequence reads were assembled using the ALLPATHS software ${ }^{40}$. This draft assembly was referred to as Parus_major 1.0.1. This version has been gap filled and cleaned of contaminating contigs. The assembly is made up of a total of 2,066 scaffolds with an N50 scaffold length of over $7.7 \mathrm{Mb}$ (N50 contig length was $133 \mathrm{~kb}$ ). The total assembled contigs spans $1.0 \mathrm{~Gb}$, and has an assembled coverage of $40 \times$ using 
fragment reads aligned to the assembly. The final assembly (Pmajor1.04) has been deposited at DDBJ/EMBL/GenBank under the accession JRXK00000000. The version described in this paper is version JRXK01000000. The genome is also publicly available in https://genomes.bioinf.nioo.knaw.nl/.

Assembly chromosome builds. Assembled scaffolds were assigned to specific chromosomes using two Parus major linkage maps ${ }^{19}$ constructed from different populations; one at Wytham Woods, Oxford (UK) and the other at de Hoge Veluwe (Netherlands), which is the population that the reference bird descended from. Flanking sequences of single-nucleotide polymorphisms (SNPs) positioned on the linkage map were aligned against the assembled scaffolds using BLAT. Scaffolds that contained multiple SNP markers were then oriented and positioned on the basis of the positions of the SNP markers on the linkage map. The order of the SNP markers on the scaffolds and the linkage map were in good agreement (Supplementary Fig. 6). Regardless of whether the Netherlands or UK map was used, the Spearman rank correlation coefficient between the linkage map marker order and the assembly marker order was 0.99 or greater for nearly all the chromosomes. Exceptions were only on microchromosomes with just a few markers, for example, linkage group 25LG22. If anything, correlation coefficients were slightly higher when the UK map was used, despite the genome assembly being performed on a Netherlands bird. This indicates that the assembly is equally applicable to other great tit populations as it is to the 'source' population. Most discrepancies between the orders on the sequence and linkage map were caused by the lower resolution of the linkage map involving SNPs that were less than $1 \mathrm{cM}$ apart. Two scaffolds (22 and 28) appeared to be chimeric and were manually split between contigs Contig22.251-Contig22.250 and contigs Contig28.53-Contig28.51, respectively. Because of a lack of any genetic marker on Contig28.52, this contig was not assigned to a specific chromosome.

Small scaffolds that were assigned to a chromosome based on only a single marker were oriented based on the zebra finch-great tit comparative map, taking into account the orientation of the flanking scaffolds assigned to that chromosome. Next, we used MUMMER to align all unassigned scaffolds against the zebra finch genome $\mathrm{e}^{20}$ and larger scaffolds that unambiguously mapped to a specific region of the zebra finch genome at a location between assigned scaffolds were assigned to that location in the genome. The orientation of these scaffolds was chosen in relation to the adjacent mapped scaffolds, thus minimizing the number of rearrangements. In total, 300 scaffolds could be assigned to a chromosomal location based on the linkage map ${ }^{19}$ totalling $975,330,736$ bp and 124 scaffolds were assigned to a chromosomal location based on the alignment with the zebra finch genome, totalling 23,489,268 bp (Supplementary Data 1). Although the final genome assembly therefore is not completely de novo, these contigs only represent $2.4 \%$ of the assembled sequence and $97.6 \%$ of the assembly represents a truly de novo genome assembly.

RNA sequencing and assembly. RNA was extracted from eight tissues (bone marrow, homogenized half of the brain, breast filet, higher intestine, kidney, liver, lung and testis) from the reference bird and was then used to prepare tissue-specific tagged Illumina sequencing libraries. The tagged libraries were pooled and sequenced using five lanes on one flowcell of Illumina HiSeq 2000 (same run on the machine). This resulted in 100 bp paired-end unstranded RNA sequencing data. The number of reads per tissue ranged from 98 to 229 million with a total number of 1.25 billion paired-end reads (Supplementary Data 7). The sequence reads were checked for quality with FastQC (http://www.bioinformatics.babraham.ac.uk/projects/fastqc/) and low-quality sequences were trimmed with Fastq-Mcf ${ }^{41}$ resulting in a final number of 1,096,140,415 paired-end reads that were used for the annotation. The RNA sequencing data per individual tissue has been submitted to Genbank (GT_BoneMarrow SRS863935, GT_Brain SRS866013, GT_BreastFilet SRS86603, GT_HigherIntestine SRS866033, GT_Liver SRS866035, GT_Kidney SRS866036, GT_Lung SRS866044, GT Testis SRS866048).

The combined 1 billion reads from all eight tissues were simultaneously de novo assembled using the Trinity software package ${ }^{42}$ v. r2013-02-25. Because of the high depth of the RNA sequencing data, we first normalized the data. Following the normalization, the Trinity assembly was subsequently run using the default settings. We obtained a total of 101,289 assembled transcripts ranging in size from 201 to $16,061 \mathrm{bp}$, with an average size of $1,335 \mathrm{bp}$. We also did a reference-based RNA assembling by using the normalized RNA sequencing data in Tophat version v2.0.10 (ref. 43; Bowtie v2.1.0 (ref. 44)).

\section{Genome annotation. For the genome annotation, both PASA v. 2-r20130605} (ref. 45) and MAKER v. 2.31 .5 (ref. 46) pipelines were used (Supplementary Fig. 7). First PASA (program to assemble spliced alignments) was used for the identification of spliced transcripts and the grouping of the identified transcripts belonging to the same gene. PASA was run using the assembled Trinity transcripts and the Cufflinks gtf output file from Tophat/Bowtie as input. Alignment within PASA was done using gmap. The PASA analysis resulted in a total of 74,229 different assembled transcripts with an average size of $1,564 \mathrm{bp}$. A comparison (using blast) with the annotated transcriptomes of chicken ${ }^{21}$, zebra finch ${ }^{20}$, flycatcher ${ }^{47}$ and ground $\mathrm{tit}^{48}$ (derived from Ensembl release 75 or NCBI refseq release 66) indicated that these transcripts represented 13,626 different genes in these other birds.
In the MAKER pipeline, the output of PASA was used as EST evidence. From the $a b$ initio predictors, AUGUSTUS version 3.0.2 (ref. 49) was applied by using the chicken gene model. The same RNA-seq de novo assembly as used in PASA was included in MAKER. In addition to de novo assembly, reference-based RNA-seq assembly was used. Last, protein evidence from zebra finch (version 3.2.4.75) and chicken (version 4.75) obtained from Ensembl version 75 was aligned to the great tit genome. By combining these two pipelines, we obtained 21,057 transcripts for 13,036 great tit genes. See additional information about functional annotation and repeat/RNA masking in Supplementary Information and Supplementary Data 8.

Resequencing and SNP calling. We analysed 29 wild great tit individuals (Supplementary Data 2) covering a wide range of the species distribution (Fig. 1); 10 individuals were from the Wytham population in Oxford (UK), and the remaining 19 birds were sampled from 15 European populations. Each bird was sequenced to $\approx 10 \times$ coverage. Paired-end sequencing libraries of each sample were built with an insert size of $300 \mathrm{bp}$ and sequencing was performed on a HiSeq 2000 platform with a read length of $100 \mathrm{bp}$. The raw reads were trimmed and filtered with Sickle (https://github.com/najoshi/sickle) using default parameters and a length restriction of 75 . We then used the Burrows-Wheeler Aligner ${ }^{50}$ to map the filtered raw reads onto the assembled great tit reference genome. Subsequently, we removed duplicates and conducted local realignments following the best practices of the GATK pipeline ${ }^{51}$. We used ANGSD ${ }^{52}$ to call SNPs based on the genotype likelihoods estimated by the GATK model from the mapped reads ${ }^{51}$; this approach has been shown to produce more accurate estimates of the Site Frequency Spectrum (SFS) than other widely used SNP-calling pipelines when sequencing coverage is low $(<10 \times)^{53}$, which is critical for our analysis because our average coverage is $10 \times$. However, for comparison, we also called SNPs using three additional approaches: (1) GATK using data from each individual separately (the 'Single' approach); (2) GATK using data from all individuals simultaneously (the 'Multi' approach); (3) Platypus, which calls SNPs directly from the Burrows-Wheeler Aligner alignments, independent of the GATK pipeline ${ }^{54}$. We also tested the reliability of the SNP data, see details in Supplementary Information and Supplementary Data 9 and 10 and Supplementary Fig. 9.

Population differentiation and demography. We found very little differentiation among populations $\left(F_{\mathrm{ST}}<0.015\right.$; Supplementary Data 3$)$. The most differentiated population pairs were Spain-UK (ES versus WW, UK; Fig. 1) and France-Spain (FR-ES; Fig. 1) with $F_{\mathrm{ST}}=0.012$

We analysed the demographic history with pairwise sequential Markovian coalescent analysis $\left(\right.$ PSMC $\left.^{23}\right)$. PSMC estimates rates of coalescent events across a single genome and uses these to infer $N_{\mathrm{e}}$ (the effective population size) in the past $^{23}$. The model relies significantly on confidently called polymorphic sites and requires that both alleles are called. Therefore we conducted this analysis with variants called on the high coverage reference genome sequence. We set the mutation rate to $2.0 \times 10^{-9}$ per year per site and the generation time to 2 years.

Genome-wide diversity and Tajima's D. We obtained genome-wide sliding window estimates (step size $10 \mathrm{~kb}$, window size $50 \mathrm{~kb}$ ) of Watterson's $\Theta$ (Fig. 2) and Tajima's $D$ (Fig. 2) along each chromosome based on the SNP calls from ANGSD. For most macrochromosomes, diversity is increased towards the chromosome ends, and there are remarkable local drops of diversity on chromosomes 3, 6 and Z (Fig. 2; more details about low diversity in chromosome Z in Supplementary Information and Supplementary Fig. 10). By calculating Watterson's $\Theta$ for synonymous sites in protein-coding genes we found a clear negative correlation between chromosome length and diversity levels (Supplementary Fig. 8); we observed the same pattern when diversity was estimated using all sites (Supplementary Fig. 8).

Selective sweep detection. We scanned the genome for target regions of positive selection using Sweepfinder ${ }^{55}$ which uses local deviations of the site frequency spectrum (SFS) compared with a standard neutral model or chromosome/genomewide reference to infer the action of positive selection. We used SNP calls from the ANGSD pipeline to construct SFS. ANGSD assumes that each variable position is biallelic and determines allele frequencies based on the genotype likelihoods ${ }^{56}$. Whole-genome alignments constructed with MAUVE ${ }^{57}$ for zebra finch ${ }^{20}$, flycatcher ${ }^{47}$, ground tit $^{48}$ and chicken ${ }^{21}$ were used to infer the ancestral state based on maximum parsimony. Sites for which the ancestral state could not be reconstructed with confidence were included in the analysis as folded sites (unpolarized). The chromosome-wide SFS was used as reference for each chromosome as recommended by Sweepfinder, which helps to reduce false positives caused by past demographic changes. We used a dense grid size of $100 \mathrm{bp}$ and extracted sweep targets that had a composite likelihood score (CLR) within the top $1 \%$. Neighbouring sweep targets were merged to sweep regions, with the total CLR score of a sweep region being the sum of the CLR score of each sweep target. The top $3 \%$ of genes that overlapped with or were near (within $5 \mathrm{kB}$ flanking the sweep region) sweep regions larger than $300 \mathrm{bp}$ were extracted (Supplementary Data 4). Targets for positive selection included the low diversity region of chromosome 3, 6 and Z. Further details of the Sweepfinder pipeline can be found in the Supplementary Materials and Methods. 


\section{Multiple alignment construction for substitution rate analyses. Natural} selection affects the composition of genomes and we were interested in estimating gene-specific rates of molecular evolution $(\mathrm{dN} / \mathrm{dS})$ and finding evidence for the action of positive selection in protein coding genes. Since alignment quality is crucial for reliably conducting tests of positive selection using substitution rate analyses, we chose a very conservative approach. We used homologous genes from zebra finch and chicken, the two best annotated bird genomes to date. We only analysed genes with a homologue in both, and used MUSCLE ${ }^{58}$ along with ZORRO $^{59}$ to exclude positions of low alignment certainty. To obtain the corresponding multiple DNA codon alignments, protein alignments along with the unaligned DNA sequences were prepared with PAL2NAL ${ }^{60}$. Altogether, we constructed $\approx 11,107$ triplet alignments. Substitution rates were calculated using PAML ${ }^{61}$ to obtain the nonsynonymous to synonymous substitution rate ratio $(\mathrm{dN} / \mathrm{dS}=\omega) . \omega$ values $<1,=1$ and $>1$ indicate purifying selection, neutral evolution and diversifying (positive) selection, respectively. Rates of molecular evolution (dN/dS) for each gene were obtained from the one-ratio model M0 from PAML that assumes a constant $\omega$ for the whole gene phylogeny. In addition, a site test was used to detect positive selection. Specifically, we compared the likelihoods calculated using model M8, which assumes a proportion of sites to evolve under positive selection, and model M7, which does not assume a site class with $\omega$ exceeding one. Positive selection was inferred when the model M7 and M8 were significantly different as assessed by a likelihood-ratio test that assumes that $2 \Delta \operatorname{lnL}$ (twice the log likelihood difference) is $\chi^{2}$ distributed with two degrees of freedom. The $P$ values were adjusted for multiple testing (Benjamini and Hochberg) with a false discovery rate of $0.2 ; \chi^{2}$ test results for an enrichment of positively selected genes were qualitatively similar for FDRs $=0.1,0.3,0.4$ and $0.5\left(P=2.17 \times 10^{-5}\right.$, $3.97 \times 10^{-3}, 2.58 \times 10^{-3}$ and $2.17 \times 10^{-3}$, respectively).

GO enrichment analyses. Human orthologues were obtained for the great tit genes by using a combination of Ensembl and Uniprot databases. In the sweep areas, orthologues were found for 460 genes. Functional relatedness of GO terms was done using the Cytoscape plugin ClueGo 2.1.4 (ref. 62). ClueGo constructs and compares networks of functionally related GO terms with kappa statistics. A two-sided hypergeometric test (enrichment/depletion) was applied with GO term fusion, network specificity was set to 'medium' and false discovery correction was carried out using the Bonferroni step-down method. We used both human (08.10.2015) and chicken gene ontologies (08.10.2015) for comparison. With human gene ontologies, we detected 13 functional groups of GO terms across all sweep areas (Supplementary Data $5 \mathrm{a}$ ). These groups were largely involved in functions concerning dendrite, cytoskeleton organization, protein oligomerization, synaptic transmission, regulation of phosphatase activity, synaptic membrane and cognition. We also did a GO enrichment analysis for the positively selected genes, as defined by the PAML-based analysis, in the same way as for the sweep genes, and obtained 12 functional GO groups (Supplementary Data $5 \mathrm{~b}$ ). When using the chicken orthologues for both sweep and positively selected genes, the results were comparable but with lower significance levels (Supplementary Data $5 c, d$ ) because the chicken genes were not as well GO annotated as the human ones.

To further confirm our GO enrichment results, we randomly selected 50 sets of genes from outside the sweep area, each containing $\sim 460$ genes (the same number as in our selective sweep set), and analysed them using the same Cluego settings. We found that the GO term groups significantly enriched in our selective sweep set appeared no more than three times except for phosphatidylinositol-mediated signalling GO group which appeared seven times. This additional analysis further supports the robustness of our results.

Test of accelerated evolution in great tit EGR1. We used BLAST to obtain orthologous sequences of the EGR1 gene from 45 additional bird species 37,38 downloaded from Ensembl version 75 or from Gigascience (http://gigadb.org/ dataset/101000, Supplementary Fig. 2, detailed species list provided in Supplementary Data 6) to test whether there was additional evidence for the action of positive selection during the evolution of EGR1 in birds. Pairwise $\mathrm{dN} / \mathrm{dS}$ rates revealed that there is a substantial increase in $\mathrm{dN} / \mathrm{dS}$ between great tit and ground tit relative to other pairwise values (Supplementary Fig. 2, upper panel). We also tested whether the increased fixations are unique to the captive reference bird by using variable positions from the 29 re-sequenced birds, the reference bird and the ground tit genome and found no evidence for this (Supplementary Fig. 2 lower panel).

Brain gene expression. To compare expression levels and methylation in the brain, 200,793,186 trimmed paired-end reads from the brain were aligned against the assembled genome using Tophat version v2.0.10 (ref. 43; Bowtie v2.1.0 (ref. 44)) with the same settings as described above, except that multiple hits were prefiltered against the genome (-M option) and the reads were first aligned against the final annotation (-G option). The brain Tophat alignment was analysed with Cufflinks v2.2.0 using the same settings as above, except that the annotation was also included (-g option). Expression levels of brain genes were extracted from the Cufflinks output.

Methylation analysis. Blood and brain DNA libraries were constructed according to the Epitect whole-genome bisulfite sequencing workflow (Illumina) with 18 PCR cycles. Whole-genome sequencing data were generated using the Illumina HiSeq 2,500 platform at Business Unit Bioscience, Wageningen UR. The number of paired-end reads ( $101 \mathrm{bp}$ ) were $358 \mathrm{M}$ and $292 \mathrm{M}$ for the brain and the blood, respectively. Raw sequencing reads were trimmed for quality $(\geq 20)$ and adaptor sequence using trim_galore v.0.1.4 (http://www.bioinformatics.babraham.ac.uk/ projects/trim_galore/). The methylation data has been submitted to NCBI with accession numbers SRR2070790 and SRR2070791 for the brain and the blood, respectively. Trimmed sequences were aligned to the reference genome using BSseeker2 v2.0.6 (ref. 63) with Bowtie2 v.2.1.0 (ref. 44) in the local alignment mode. A total, $97.63 \%$ and $99.93 \%$ of the genome was covered to an average depth of $31.88 \times$ and $33.04 \times$ in brain and blood, respectively. Methylation levels for each site were determined using the BSseeker2 methylation call script. All the analyses were done using sites covered by a minimum of 10 reads in both the samples. Only genes found to be 1:1:1 orthologues with chicken and zebra finch were used for methylation analysis. Gene bodies (annotated gene boundaries excluding the $5^{\prime} 5 \%$ of genes) and TSS ( $300 \mathrm{bp}$ upstream to $50 \mathrm{bp}$ downstream of the annotated starting position of each gene) for which we had information from at least $50 \%$ of the potential methylation sites were used in the $\mathrm{dN} / \mathrm{dS}$ and expression correlation analysis. Average methylation levels for TSS and gene bodies were calculated for each individual gene. The upper (highly methylated) and lower (lowly methylated) quartiles were compared for differences in their evolutionary rates (dN/dS) using a Mann-Whitney $U$-test. Correlations between the average methylation level of a given region (TSS or gene body) per gene with expression were performed using Spearman's rank correlation. A sliding window approach was used to infer differences in methylation levels between sweep and non-sweep gene regions. Non-sweep genes located on scaffolds were not included in the analysis, as these regions were not tested for sweeps. For this, genes were divided into different regions: the gene body (described above), $10 \mathrm{~kb}$ upstream and $10 \mathrm{~kb}$ downstream of the gene body and the TSS region (described above). Each gene body was subdivided into 40 bins, with the length of each bin therefore depending on the length of the gene. We calculated the mean methylation levels of these 40 bins with an overlap between neighbouring bins of $250 \mathrm{bp}$. Upstream and downstream regions were divided into bins of exactly $250 \mathrm{bp}$ with an overlap of $125 \mathrm{bp}$ between consecutive bins.

To compare TSS methylation between sweep and non-sweep genes, we conducted a $t$-test with equal variance assumed. Only TSS regions with a minimum of 10 covered sites were used for comparative analysis. To compare methylation levels between sweep and non-sweep genes for other gene regions, we conducted LMM analyses with methylation level as the dependent variable, sweep (yes or no) as a fixed factor and bin as a random factor. A likelihood-ratio test was conducted comparing the model with and without sweep as a fixed factor. All criteria were met for conducting parametric analyses.

\section{References}

1. Rendell, L. et al. Cognitive culture: theoretical and empirical insights into social learning strategies. Trends Cogn. Sci. 15, 68-76 (2011).

2. Leadbeater, E. What evolves in the evolution of social learning? J. Zool. 295, 4-11 (2015).

3. Aplin, L. M. et al. Experimentally induced innovations lead to persistent culture via conformity in wild birds. Nature 518, 538-541 (2015).

4. Titulaer, M., van Oers, K. \& Naguib, M. Personality affects learning performance in difficult tasks in a sex-dependent way. Anim. Behav. 83, 723-730 (2012).

5. Cole, E. F., Morand-Ferron, J., Hinks, A. E. \& Quinn, J. L. Cognitive ability influences reproductive life history variation in the wild. Curr. Biol. 22, 1808-1812 (2012).

6. Overington, S. E., Morand-Ferron, J., Boogert, N. J. \& Lefebvre, L. Technical innovations drive the relationship between innovativeness and residual brain size in birds. Anim. Behav. 78, 1001-1010 (2009).

7. Boyce, M. S. \& Perrins, C. M. Optimizing great tit clutch size in a fluctuating environment. Ecology 68, 142-153 (1987).

8. Nussey, D. H., Postma, E., Gienapp, P. \& Visser, M. E. Selection on heritable phenotypic plasticity in a wild bird population. Science 310, 304-306 (2005).

9. Charmantier, A. et al. Adaptive phenotypic plasticity in response to climate change in a wild bird population. Science 320, 800-803 (2008).

10. Pettifor, R. A., Perrins, C. M. \& McCleery, R. H. Individual optimization of clutch size in great tits. Nature 336, 160-162 (1988).

11. Knowles, S. C. L., Nakagawa, S. \& Sheldon, B. C. Elevated reproductive effort increases blood parasitaemia and decreases immune function in birds: a meta-regression approach. Funct. Ecol. 23, 405-415 (2009).

12. Bouwhuis, S., Sheldon, B. C., Verhulst, S. \& Charmantier, A. Great tits growing old: selective disappearance and the partitioning of senescence to stages within the breeding cycle. Proc. R. Soc. B Biol. Sci. 276, 2769-2777 (2009).

13. Van Noordwijk, A. J. \& Scharloo, W. Inbreeding in an island population of the great tit. Evolution 35, 674-688 (1981).

14. Greenwood, P. J., Harvey, P. H. \& Perrins, C. M. Inbreeding and dispersal in the great tit. Nature 271, 52-54 (1978).

15. Richner, H. Host-parasite interactions and life-history evolution. Zoology 101, 333-344 (1998). 
16. Krebs, J. R. Territory and breeding density in the great tit, Parus major $\mathrm{L}$. Ecology 52, 2-22 (1971).

17. Mappes, J. \& Alatalo, R. V. Effects of novelty and gregariousness in survival of aposematic prey. Behav. Ecol. 8, 174-177 (1997).

18. Van Oers, K. \& Naguib, M. in Animal Personalities: Behavior, Physiology and Evolution (eds Carere, C. \& Maestripieri, D.) 520 (Chicago Univ. Press, 2013).

19. Van Oers, K. et al. Replicated high-density genetic maps of two great tit populations reveal fine-scale genomic departures from sex-equal recombination rates. Heredity 112, 307-316 (2014).

20. Warren, W. C. et al. The genome of a songbird. Nature 464, 757-762 (2010).

21. ICGSC. Sequence and comparative analysis of the chicken genome provide unique perspectives on vertebrate evolution. Nature 432, 695-716 (2004).

22. Kvist, L. et al. Evolution and genetic structure of the great tit (Parus major) complex. Proc. R. Soc. B Biol. Sci. 270, 1447-1454 (2003).

23. Li, H. \& Durbin, R. Inference of human population history from individual whole-genome sequences. Nature 475, 493-496 (2011).

24. Clayton, D. F. The genomics of memory and learning in songbirds. Annu. Rev. Genomics Hum. Genet. 14, 45-65 (2013).

25. Hara, E., Kubikova, L., Hessler, N. A \& Jarvis, E. D. Role of the midbrain dopaminergic system in modulation of vocal brain activation by social context. Eur. J. Neurosci. 25, 3406-3416 (2007).

26. Dragunow, M. A role for immediate-early transcription factors in learning and memory. Behav. Genet. 26, 293-299 (1996).

27. Bolhuis, J. J., Zijlstra, G. G. O., den Boer-Visser, A. M. \& Van Der Zee, E. A. Localized neuronal activation in the zebra finch brain is related to the strength of song learning. Proc. Natl Acad. Sci. USA 97, 2282-2285 (2000).

28. Enard, W. et al. Molecular evolution of FOXP2, a gene involved in speech and language. Nature 418, 869-872 (2002).

29. Teramitsu, I. \& White, S. A. FoxP2 regulation during undirected singing in adult songbirds. J. Neurosci. 26, 7390-7394 (2006).

30. Haesler, S. et al. Incomplete and inaccurate vocal imitation after knockdown of FoxP2 in songbird basal ganglia nucleus area X. PLoS Biol. 5, e321 (2007).

31. Goldberg, A. D., Allis, C. D. \& Bernstein, E. Epigenetics: a landscape takes shape. Cell 128, 635-638 (2007).

32. Ball, M. P., Li, J. B., Gao, Y., Lee, J. \& Leproust, E. Targeted and genome-scale methylomics reveals gene body signatures in human cell lines. Nat. Biotechnol. 27, 361-368 (2009).

33. Guo, J. U. et al. Distribution, recognition and regulation of non-CpG methylation in the adult mammalian brain. Nat. Neurosci. 17, 215-222 (2014).

34. Shirane, K. et al. Mouse oocyte methylomes at base resolution reveal genome-wide accumulation of non-CpG methylation and role of DNA methyltransferases. PLoS Genet. 9, e1003439 (2013).

35. Lister, R. et al. Human DNA methylomes at base resolution show widespread epigenomic differences. Nature 462, 315-322 (2009).

36. Nätt, D. et al. Heritable genome-wide variation of gene expression and promoter methylation between wild and domesticated chickens. BMC Genomics 13, 59 (2012).

37. Jarvis, E. D. et al. Whole-genome analyses resolve early branches in the tree of life of modern birds. Science 346, 1320-1331 (2014).

38. Zhang, G. et al. Comparative genomics reveals insights into avian genome evolution and adaptation. Science 346, 1311-1320 (2014).

39. Drent, P. J., van Oers, K. \& van Noordwijk, A. J. Realized heritability of personalities in the great tit (Parus major). Proc. R. Soc. B Biol. Sci. 270, 45-51 (2003).

40. Gnerre, S. et al. High-quality draft assemblies of mammalian genomes from massively parallel sequence data. Proc. Natl. Acad. Sci. USA 108, 1513-1518 (2011).

41. Aronesty, E. Comparison of sequencing utility programs. Open Bioinforma. J. 7, 1-8 (2013).

42. Grabherr, M. G. et al. Full-length transcriptome assembly from RNA-Seq data without a reference genome. Nat. Biotechnol. 29, 644-652 (2011).

43. Kim, D. et al. TopHat2: accurate alignment of transcriptomes in the presence of insertions, deletions and gene fusions. Genome Biol. 14, R36 (2013).

44. Langmead, B., Trapnell, C., Pop, M. \& Salzberg, S. L. Ultrafast and memoryefficient alignment of short DNA sequences to the human genome. Genome Biol. 10, R25 (2009).

45. Haas, B. J. et al. De novo transcript sequence reconstruction from RNA-seq using the Trinity platform for reference generation and analysis. Nat. Protoc. 8, 1494-1512 (2013).

46. Cantarel, B. L. et al. MAKER: an easy-to-use annotation pipeline designed for emerging model organism genomes. Genome Res. 18, 188-196 (2008).

47. Ellegren, H. et al. The genomic landscape of species divergence in Ficedula flycatchers. Nature 491, 756-760 (2012).

48. Qu, Y. et al. Ground tit genome reveals avian adaptation to living at high altitudes in the Tibetan plateau. Nat. Commun. 4, 2071 (2013).

49. Stanke, M., Tzvetkova, A. \& Morgenstern, B. AUGUSTUS at EGASP: using EST, protein and genomic alignments for improved gene prediction in the human genome. Genome Biol. 7(Suppl 1): S11.1-8 (2006).
50. Li, H. \& Durbin, R. Fast and accurate short read alignment with BurrowsWheeler transform. Bioinformatics 25, 1754-1760 (2009).

51. DePristo, M. A. et al. A framework for variation discovery and genotyping using next-generation DNA sequencing data. Nat. Genet. 43, 491-498 (2011).

52. Korneliussen, T., Albrechtsen, A. \& Nielsen, R. ANGSD: Analysis of Next Generation Sequencing Data. BMC Bioinformatics 15, 356 (2014).

53. Han, E., Sinsheimer, J. S. \& Novembre, J. Characterizing bias in population genetic inferences from low-coverage sequencing data. Mol. Biol. Evol. 31, 723-735 (2014).

54. Rimmer, A. et al. Integrating mapping-, assembly- and haplotype-based approaches for calling variants in clinical sequencing applications. Nat. Genet 46, 912-918 (2014).

55. Nielsen, R. et al. Genomic scans for selective sweeps using SNP data. Genome Res. 15, 1566-1575 (2005).

56. Kim, S.Y. et al. Estimation of allele frequency and association mapping using next-generation sequencing data. BMC Bioinformatics 35, 231 (2011).

57. Darling, A. E., Mau, B. \& Perna, N. T. progressiveMauve: multiple genome alignment with gene gain, loss and rearrangement. PLoS ONE 5, el1147 (2010).

58. Edgar, R. C. MUSCLE: multiple sequence alignment with high accuracy and high throughput. Nucleic Acids Res. 32, 1792-1797 (2004).

59. Wu, M., Chatterji, S. \& Eisen, J. A. Accounting for alignment uncertainty in phylogenomics. PLoS ONE 7, e30288 (2012).

60. Suyama, M., Torrents, D. \& Bork, P. PAL2NAL: Robust conversion of protein sequence alignments into the corresponding codon alignments. Nucleic Acids Res. 34, W609-W612 (2006).

61. Yang, Z. PAML 4: phylogenetic analysis by maximum likelihood. Mol. Biol. Evol. 24, 1586-1591 (2007).

62. Bindea, G. et al. ClueGO: A Cytoscape plug-in to decipher functionally grouped gene ontology and pathway annotation networks. Bioinformatics 25, 1091-1093 (2009).

63. Guo, W. et al. BS-Seeker2: a versatile aligning pipeline for bisulfite sequencing data. BMC Genomics 14, 774 (2013).

\section{Acknowledgements}

We thank Eveline Verhulst for help with the methylome data, Christa Mateman for lab assistance, Martijn Derks for calculating the sliding windows, Tieshan Xu for the help with the Trinity assembly, Louise Dittmar for the help in $\mathrm{dN} / \mathrm{dS}$ and diversity analysis, Christian Huber for help on the sweep analysis and Jun-Mo Kim who designed the SNP chip. K.M.S. was supported by a grant from the Cooperative Research Program for Agriculture Science \& Technology Development (PJ009103) of the Rural Development Administration, Republic of Korea. T.I.G., P.C. and K.Z. were supported by a BBSRC grant (BB/K000209/1) and a NERC grant (NE/L005328/1) awarded to K.Z., C.J.G. was funded by Natural Environment Research Council (NERC) (NE/K01126X/1). K.J.F.V. was funded by the Dutch Organisation for Scientific Research, NWO VIDI grant (864.10.008). B.C.S. was funded by ERC Advanced Grant (250164) and by a Wolfson Merit Award from the Royal Society. J.S. was funded by a European Research Council (ERC) Starting grant, Avian EGG (202487) and a Natural Environment Research Council (NERC), The Great Tit HapMap Project (NE/J012599/1). M.E.V. was supported by the Netherlands Organisation for Scientific Research (NWO-VICI grant) and the European Research Council (ERC-2013-AdG 339092).

\section{Author contributions}

M.A.M.G., M.E.V. and K.v.O. designed the research. P.M., W.C.W. and M.A.M.G. did the genome assembly. V.N.L., V.d.J. and H.J.M. did the genome annotation. R.P.M.A.C isolated DNA and RNA and coordinated RNA sequencing. T.I.G., P.C. and K.Z. performed and coordinated the diversity and signatures of selection analyses. C.J.G. did the effective population size analyses. O.M. coordinated the methylome analysis and performed the gene expression analyses. K.v.O. and K.J.F.V. initiated the methylome sequencing and K.v.O., K.M.S., O.M., K.J.F.V. and T.I.G. analysed the methylome data. B.C.S., K.v.O., M.E.V. and TGTHC provided great tit DNA samples and J.S. coordinated the re-sequencing of additional birds. J.S., K.v.O. and M.A.M.G. performed the comparison between the linkage maps and genome assembly. V.N.L. did the GO analyses. V.N.L., T.I.G., K.M.S., C.J.G., K.v.O. and M.A.M.G. wrote the manuscript. All the authors provided input during the writing of the manuscript.

\section{Additional information}

Accession Codes: The raw sequences of reference great tit have been deposited to the NCBI Sequence Read Archive (SRA; http://www.ncbi.nlm.nih.gov/sra) under the accession number SRS1185780. The final assembly (Pmajor1.04) has been deposited in DDBJ/EMBL/GenBank under the accession JRXK00000000. The version described in this paper is version JRXK01000000. The RNAseq reads for the eight tissues have been deposited to the NCBI Sequence Read Archive under the accession number GT_BoneMarrow SRS863935, GT_Brain SRS866013, GT_BreastFilet SRS866031, GT_HigherIntestine SRS866033, GT_Liver SRS866035, GT_Kidney SRS866036, GT_Lung SRS866044, GT_Testis SRS866048. The whole-genome bisulfite sequencing reads for the two tissues have been deposited to the NCBI Sequence Read Archive under 
the accession numbers GT_Brain_BS SRS964344 and GT_Blood_BS SRS964345. The reads for the 29 re-sequenced birds have been deposited to the NCBI Sequence Read Archive under the accession number SRP066678.

Supplementary Information accompanies this paper at http://www.nature.com/ naturecommunications

Competing financial interests: The authors declare no competing financial interests.

Reprints and permission information is available online at http://npg.nature.com/ reprintsandpermissions/
How to cite this article: Laine, V. N. et al. Evolutionary signals of selection on cognition from the great tit genome and methylome. Nat. Commun. 7:10474 doi: 10.1038/ncomms10474 (2016).

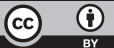

This work is licensed under a Creative Commons Attribution 4.0 International License. The images or other third party material in this article are included in the article's Creative Commons license, unless indicated otherwise in the credit line; if the material is not included under the Creative Commons license, users will need to obtain permission from the license holder to reproduce the material. To view a copy of this license, visit http://creativecommons.org/licenses/by/4.0/

\section{The Great Tit HapMap Consortium}

\section{Frank Adriaensen ${ }^{8}$, Eduardo Belda9 ${ }^{9}$ Andrey Bushuev ${ }^{10}$, Mariusz Cichon ${ }^{11}$, Anne Charmantier ${ }^{12}$, Niels Dingemanse ${ }^{13}$, Blandine Doligez ${ }^{14}$, Tapio Eeva ${ }^{15}$, Kjell Einar Erikstad ${ }^{16}$, Slava Fedorov ${ }^{17}$, Michaela Hau ${ }^{13}$, Sabine Hille ${ }^{18}$, Camilla Hinde ${ }^{19}$, Bart Kempenaers ${ }^{13}$, Anvar Kerimov ${ }^{10}$, Milos Krist ${ }^{20}$, Raivo Mand ${ }^{21}$, Erik Matthysen ${ }^{8}$, Reudi Nager ${ }^{22}$, Claudia Norte ${ }^{23}$, Markku Orell ${ }^{24}$, Heinz Richner ${ }^{25}$, Tore Slagsvold ${ }^{26}$, Vallo Tilgar ${ }^{21}$, Joost Tinbergen ${ }^{27}$, Janos Torok $^{28}$, Barbara Tschirren ${ }^{29}$, Tera Yuta ${ }^{30}$}

${ }^{8}$ Evolutionary Ecology Group, Department of Biology, University of Antwerp, B-2020 Antwerp, Belgium. ${ }^{9}$ Departamento de Ciencia Animal, IGIC, Universidad Politécnica de Valencia, C/Paranimf n¹, E-46730 Gandía (Valencia), España. ${ }^{10}$ Faculty of Biology, Lomonosov Moscow State University, Moscow 119234, Russia. ${ }^{11}$ Inst. of Environmental Sciences, Jagiellonian University, Gronostajowa 7, 30-387 Kraków, Poland. ${ }^{12}$ CEFE-CNRS, UMR 5175, 1919, route de Mende, F34293 Montpellier Cedex 5, France. ${ }^{13}$ Max Planck Institute for Ornithology, Department of Behavioural Ecology \& Evolutionary Genetics, EberhardGwinner-Straße, House 5, 82319 Seewiesen (Starnberg), Germany. ${ }^{14}$ UMR CNRS 5558-LBBE, Biométrie et Biologie Évolutive, UCB Lyon 1 - Bât. Grégor Mendel, 43 bd du 11 novembre 1918, 69622 VILLEURBANNE cedex, France. ${ }^{15}$ Section of Ecology, Department of Biology, University of Turku, Turku 20014 , Finland. ${ }^{16}$ Norwegian Institute for Nature Research, FRAM-High North Research Centre for Climate and the Environment, 9296 Tromsø, Norway.

${ }^{17}$ Department of Vertebrate Zoology, Moscow State University, Moscow 119899 Russia. ${ }^{18}$ Institute of Wildlife Biology and Game Management, University of Natural Resources and Life Science, A-1180 Vienna, Austria. ${ }^{19}$ Behavioural Ecology Group, Department of Animal Sciences, Wageningen University,

Wageningen 6708 PB, The Netherlands. ${ }^{20}$ Department of Zoology and Laboratory of Ornithology, Faculty of Science, Palacký University, Olomouc 77147, Czech Republic. ${ }^{21}$ Department of Zoology, Institute of Ecology and Earth Sciences, University of Tartu, Vanemuise 46, Tartu 51014, Estonia. 22Institute of Biodiversity, Animal Health and Comparative Medicine, University of Glasgow, Glasgow G12 8QQ, UK. ${ }^{23}$ Department of Life Sciences, Institute of Marine Research IMAR/CMA, University of Coimbra, Coimbra, Portugal. ${ }^{24}$ Department of Biology, University of Oulu, P.O. Box 3000, 90014 Oulu, Finland. ${ }^{25}$ Evolutionary Ecology Lab, Institute of Ecology and Evolution, University of Bern, Bern 3012, Switzerland. ${ }^{26}$ Centre for Ecological and Evolutionary Synthesis (CEES), Department of Biosciences, University of Oslo, P.O. Box 1066, Blindern, 0316 Oslo, Norway. ${ }^{27}$ Centre for Ecological and Evolutionary Studies (CEES), Univ. of Groningen, PO Box 11103, NL-9700 CC Groningen, The Netherlands. ${ }^{28}$ Behavioural Ecology Group, Department of Systematic Zoology and Ecology, Eötvös Loránd University, Budapest H-1117, Hungary. ${ }^{29}$ Institute of Evolutionary Biology and Environmental Studies, University of Zurich, Winterthurerstrasse 190, CH-8057 Zurich, Switzerland. ${ }^{30}$ Graduate School of Environmental Science, Hokkaido University, N10 W5 Sapporo, Hokkaido 060-0810, Japan. 